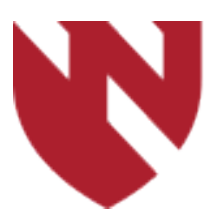

December 2019

\title{
A Retrospective Study at Two Level One Trauma Centers on the Association of Internal Injuries with Femoral Fractures
}

\author{
Ryan E. Miller \\ University of Nebraska Medical Center \\ Catherine Olinger \\ University of Tennessee Health Science Center \\ Leonid Grossman \\ University of Pittsburgh \\ Dennis Chakkalakal \\ Creighton University \\ Karl Bergmann \\ Creighton University
}

See next page for additional authors

Tell us how you used this information in this short survey.

Follow this and additional works at: https://digitalcommons.unmc.edu/gmerj

Part of the Higher Education Commons, and the Medicine and Health Sciences Commons

\section{Recommended Citation}

Miller, R. E., Olinger, C., Grossman, L., Chakkalakal, D., Bergmann, K., Lyden, E., , Siebler, J. A Retrospective Study at Two Level One Trauma Centers on the Association of Internal Injuries with Femoral Fractures. Graduate Medical Education Research Journal. 2019 Dec 13; 1(1).

https://digitalcommons.unmc.edu/gmerj/vol1/iss1/2

This Original Report is brought to you for free and open access by DigitalCommons@UNMC. It has been accepted for inclusion in Graduate Medical Education Research Journal by an authorized editor of DigitalCommons@UNMC. For more information, please contact digitalcommons@unmc.edu. 


\title{
A Retrospective Study at Two Level One Trauma Centers on the Association of Internal Injuries with Femoral Fractures
}

\author{
Abstract \\ Abstract: Injuries capable of fracturing the femur often involve concurrent internal organ damage. \\ However, up to $25 \%$ of injuries are initially missed. Prior studies evaluating the association of femur \\ fractures with internal injury included only automobile trauma, were skewed toward more severe injuries, \\ and were broad database studies. To our knowledge, there are no studies of this kind that include bicycle, \\ motorcycle, and motor vehicle-pedestrian trauma, excluding those deceased at the scene, and which \\ included chart reviews. We hypothesized that in the trauma setting, the presence of a femur fracture \\ would correlate with an increase in concomitant internal organ injuries. Data was retrospectively queried \\ from two Level I Trauma Centers. Patients presenting between January 1, 2005 and December 31, 2012 \\ with trauma activation met inclusion criteria. Patients were stratified based on presence of a femur \\ fracture, open/closed fracture status, and shaft versus non-shaft fractures. Internal organ injuries were \\ documented. Logistic regression analysis was used to determine if the presence of a femur fracture, open \\ fractures, or shaft fractures were predictive of internal injuries. Results were reported as odds ratios with \\ $95 \%$ confidence intervals $(\mathrm{Cl})$. A p-value $<0.05$ was statistically significant. Femur fracture and open \\ fracture were associated with additional internal injury. Shaft fractures were not associated with \\ additional injury. Subjects $\leq 18$ years with femur fracture were more likely to sustain additional injury \\ compared to older age groups. The current study reveals that fractures of the femur in this setting may be \\ associated with additional internal injuries. Open fractures may portend more severe organ injury \\ compared to closed fractures. Femoral fracture in age $\geq 65$ may not be as predictive for associated \\ internal injuries. Development of a standardized grading system may aid in alerting the provider to the \\ potential for these life-threatening injuries. Level of evidence: IV.
}

\section{Keywords}

femoral, femur, fracture, internal injury, organ, trauma

\section{Creative Commons License}

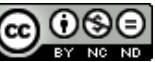

This work is licensed under a Creative Commons Attribution-Noncommercial-No Derivative Works 4.0 License.

\section{Authors}

Ryan E. Miller, Catherine Olinger, Leonid Grossman, Dennis Chakkalakal, Karl Bergmann, Elizabeth Lyden, and Justin Siebler 


\section{A Retrospective Study at Two Level One Trauma Centers on the Association of Internal Injuries with Femoral Fractures}

Ryan E. Miller ${ }^{1}$, Catherine Olinger ${ }^{2}$, Leonid Grossman ${ }^{3}$, Dennis Chakkalakal ${ }^{4}$, Karl Bergmann ${ }^{4}$, Elizabeth Lyden ${ }^{5}$, Justin Siebler ${ }^{1}$

${ }^{1}$ University of Nebraska Medical Center, Department of Orthopaedic Surgery

${ }^{2}$ University of Tennessee Health Science Center, Department of Orthopaedic Surgery

${ }^{3}$ University of Pittsburgh, Department of Orthopaedic Surgery

${ }^{4}$ Creighton University, School of Medicine, Division of Orthopaedic Surgery

${ }^{5}$ University of Nebraska Medical Center, College of Public Health

https://doi.org/10.32873/unmc.dc.gmerj.1.1.002

\begin{abstract}
Introduction: Injuries capable of fracturing the femur often involve concurrent internal organ damage. However, up to $25 \%$ of injuries are initially missed. Prior studies evaluating the association of femur fractures with internal injury included only automobile trauma, were skewed toward more severe injuries, and were broad database studies. To our knowledge, there are no studies of this kind that include bicycle, motorcycle, and motor vehicle-pedestrian trauma, excluding those deceased at the scene, and which included chart reviews. We hypothesized that, in the trauma setting, the presence of a femur fracture correlates with an increase in concomitant internal organ injuries.
\end{abstract}

Methods: Data were retrospectively queried from two Level I Trauma Centers. Patients presenting between January 1, 2005 and December 31, 2012 with trauma activation met inclusion criteria. Patients were stratified based on presence of a femur fracture, open/ closed fracture status, and shaft versus nonshaft fractures. Internal organ injuries were documented. Logistic regression analysis was used to determine if the presence of a femur fracture, open fractures, or shaft fractures were predictive of internal injuries. Results were reported as odds ratios with $95 \%$ confidence intervals (CI). A p-value $<0.05$ was statistically significant.

Results: Femur fracture and open fracture were associated with additional internal injury. Shaft fractures were not associated with additional injury. Subjects' $\leq 18$ years of age with femur fracture were more likely to sustain additional injury compared to older age groups.

Conclusion: The current study reveals that fractures of the femur in this setting may be associated with additional internal injuries. Open fractures may portend more severe organ injury compared to closed fractures. Femoral fracture in age $\geq 65$ may not be as predictive for associated internal injuries. Development of a standardized grading system may aid in alerting the provider to the potential for these life-threatening injuries.

\section{Introduction}

Femoral shaft fractures occur with average incidence of 10 per 100,000 person-years. ${ }^{1}$ Epidemiologic studies demonstrate a bimodal distribution, with a propensity for higher energy injuries in the young male population and low energy ground level falls in the older female population. The most common cause for high energy femoral shaft fractures is motor vehicle accident. ${ }^{2}$ As the largest and strongest bone in the body, with an isolated fracture load of 5-51 joules, trauma capable of fracturing the femur should prompt providers to seek concurrent injuries. ${ }^{3}$ Several studies have asserted that femur fractures are associated with higher Injury Severity Index (ISS) and injury to internal organs. ${ }^{4,5}$ However, due to their distracting and often dramatic appearance, up to one in four associated injuries may go undiagnosed and untreated, leading to delayed care. ${ }^{6,7}$ Nikolaou et al. reported increasing fracture severity was predictive of concomitant thoracic, pelvic, and other extremity injuries. ${ }^{8}$ However, these studies included only automobile trauma and were skewed toward more severe injuries. ${ }^{4,9}$ Similar studies have demonstrated injury associations. Adili et al. showed an association of humerus fractures in motor vehicle collisions with other musculoskeletal injury, as well as liver injuries. ${ }^{5}$ Sheyerer et al. demonstrated an association of fractures of the sternum and other chest and spine injuries. ${ }^{10}$ Recently, Anandasivam et al. surveyed 26,357 adults with femoral shaft fractures in the National Trauma Data Bank (NTDB) between 2011 and 2012. They demonstrated a significant association of femoral shaft fractures and internal organ injury. The top three associated injuries included: lung (18.9\%), intra-cranial (13.5\%), and liver $(6.2 \%)$ injuries. ${ }^{11}$ We sought to investigate associations of femoral fracture with internal injuries at two, local, Level 1 Trauma Centers with a large regional referral network. We hypothesized that femoral fracture, open fracture, and diaphyseal femoral fracture would be predictive of associated internal injury.

\section{Methods}

Data were retrospectively queried from the Trauma Registry of the American College of Surgeons (TRACS) at two Level I Trauma Centers. The study was approved by the Institutional Review Board (IRB) from both centers. Patients presenting with trauma activation, including motor vehicle, motorcycle, bicycle, and pedestrian versus motor vehicle mechanisms between January 1, 2005 and December 31, 2012 met inclusion criteria. Patients presenting with either a gunshot wound or ground level fall were excluded. Demographic information, including age and gender, as well as cause of injury, co-morbidities, injuries, and treatment provided was recorded. Internal injuries were stratified by anatomic location - thoracic (pulmonary contusion, cardiac, pneumothorax, diaphragm injury, bronchial tree), abdominal (spleen, liver, intestine, kidney, pancreas), and pelvic (ureter, urethra, vascular). Patients were stratified based on the presence or absence of a femur fracture, as well as open/closed status of the fracture. Femoral fracture type (head, neck, intertrochanteric, subtrochanteric, shaft, distal, and other) was recorded from one center.

A two sample t-test was used to compare age and the presence of individual, internal injuries. Associations between categorical variables (e.g. age group and fracture group; open/closed status and internal injuries) were analyzed with Fisher's exact test. The predictive value of a femur fracture and any association with internal injuries were analyzed using logistic regression. Separate logistic regression models were run for each internal injury, with femur fracture status (open/closed) and fracture pattern (shaft versus non-shaft) as the predictor. Ureter and pancreas injuries were excluded, as no subjects from the femur fracture cohort had either injury. Results were reported as odds ratios, 95\% confidence intervals and p-values (determined from logistic regression). Statistical analysis was performed using SAS 9.4 (SAS Institute Inc., Cary N.C., U.S.). P-values were adjusted for multiple testing using Benjamini-Hochberg's False Discovery Rate. A p-value $<0.05$ was considered statistically significant. 


\section{Results}

A total of 4,788 patients were included in the study. There were 530 patients (11\%) with a femoral fracture. The fracture cohort was associated with more internal injuries to the spleen (OR 1.8; 95\% CI: 1.3-2.4; $\mathrm{p}=0.002$ ), liver (OR 1.5; 95\% CI: 1.1-2.2; $\mathrm{p}=0.05$ ), intestine (OR 4; 95\% CI: 2.3-7.1; $\mathrm{p}=0.001$ ), and bronchial tree (OR 5.4; 95\% CI: 1.5-19.2; $\mathrm{p}=0.04$ ) (Table 1). For example, patients presenting with a femur fracture were 4 times as likely to have a bowel injury when compared to patients without a femur fracture. There was no association for the other observed injuries. When grouped by anatomic region, only abdominal injuries remained significantly associated with femur fractures (OR 1.6; 95\% CI: 1.3-2.1; $\mathrm{p}=0.0001$ ).

There was a statistically significant association between femur fractures and age $(\mathrm{p}=0.0037)$ (Table 2). Younger patients $(\leq 18$ years old) were more likely to sustain a femoral fracture $(14.3 \%)$ than individuals $19-64(10.5 \%)$ or seniors $(9.7 \%)$. To avoid confounding variables, a separate logistic regression model was performed to control for age, which did not appear to alter the results (Table 3 ). In a stratified analysis by age group, subjects $\leq 18$ years old within the femur fracture cohort were more likely to sustain injury to the aorta (OR 12.3; 95\% CI: 2.2-68; $\mathrm{p}=0.0039$ ), spleen (OR 1.5; 95\% CI: 1.5-4.4; $\mathrm{p}=0.0008$ ), liver (OR 2.3; 95\% CI: 1.3-4.1; $\mathrm{p}=0.0057$ ), and intestine (OR 4.9; 95\% CI: 1.3-18.6; $\mathrm{p}=0.019$ ) (Table 3). Patients 19-64 years of age also showed increased injury to spleen (OR 1.5; 95\% CI: 1.0-2.3; $\mathrm{p}=0.042$ ), liver (OR 3.0; 95\% CI: 1.3-4.1), intestine (OR 4.0; 95\% CI: 2.0-7.7; $\mathrm{p}<0.0001$ ), and bronchial tree (OR 8.6; 95\% CI: 1.1-34.8; $\mathrm{p}=0.0023)$.

Open fractures were associated an increased risk of spleen (OR 2.3; 95\% CI: 1.1-4.8; $\mathrm{p}=0.04$ ), liver (OR 2.36; 95\% CI: 1.1-5.2; $\mathrm{p}=0.04$ ), and overall abdominal (OR 2.3; 95\% CI: 1.3-4.2; $\mathrm{p}=0.01$ ) injuries compared to closed fractures (Table 4). There was no association between fracture subtype (shaft versus non-shaft) and associated injuries.

\section{Discussion}

The prior study by Adili et al. found an association between femoral fractures sustained in motor vehicle accidents and hemopneumothorax and bowel injury. ${ }^{4}$ Anandasivam et al. recently demonstrated associated injuries including: lung (18.9\%), intra-cranial (13.5\%), and liver $(6.2 \%)$ injuries in a large database study. ${ }^{11}$ In our study we
Table 1.

Femur fractures were associated with an increased incidence of injuries to the aorta, spleen, liver intestine and bronchial tree.

\begin{tabular}{lcccc} 
Internal Injury & Unadjusted Odds Ratio & 95\% Confidence Limits & P-value* \\
\hline Any Internal injury & 1.41 & 1.17 & 1.7 & 0.0003 \\
\hline Thoracic & 1.16 & 0.94 & 1.43 & 0.16 \\
\hline Abdominal & 1.61 & 1.26 & 2.06 & 0.0001 \\
\hline Pelvic & 1.54 & 0.72 & 3.3 & 0.27 \\
\hline Sub Regions & & & & 0.33 \\
\hline Pulm cont & 1.8 & 0.92 & 1.52 & 0.53 \\
\hline Cardiac & 1.35 & 0.66 & 2.7 & 0.45 \\
\hline Pneumothorax & 0.86 & 0.68 & 1.13 & 0.83 \\
\hline Diaphragm & 0.8 & 0.18 & 3.44 & 0.04 \\
\hline Bronchial tree & 5.39 & 1.52 & 19.16 & 0.08 \\
\hline Aorta & 2.2 & 1.09 & 4.44 & 0.002 \\
\hline Spleen & 1.77 & 1.3 & 2.43 & 0.05 \\
\hline Liver & 1.54 & 1.09 & 2.18 & 0.001 \\
\hline Intestine & 4.01 & 2.27 & 7.1 & 0.91 \\
\hline Bladder & 1.06 & 0.41 & 2.7 & 0.30 \\
\hline Kidney & 1.51 & 0.85 & 2.7 & 0.65 \\
\hline Urethra & 1.31 & 0.55 & 3.11 & 0.24 \\
\hline Pelvic Vasculature & 4.03 & 0.74 & 22.05 & \\
\hline -Values & & 179 & \\
\hline
\end{tabular}

*P-values for sub-regions are adjusted for multiple testing using Benjamini-Hochberg's False Discovery Rate.

\section{Table 2.}

A larger percentage of patients between the ages 0-18 presented with femur fractures than did patients between the ages $19-64$ or 65 and older (14.3\% versus $10.4 \%$ versus $9.7 \%$, respectively).

\begin{tabular}{cccc} 
Age (years) & $\begin{array}{c}\text { Negative for } \\
\text { Femur Fracture \# (\%) }\end{array}$ & $\begin{array}{c}\text { Positive for } \\
\text { Femur Fracture \# (\%) }\end{array}$ & Total \\
\hline $0-18$ & $748(85.7)$ & $125(14.3)$ & 873 \\
\hline $19-64$ & $3016(89.5)$ & $352(10.5)$ & 3368 \\
\hline $65+$ & $494(90.3)$ & $53(9.7)$ & 547 \\
\hline Total & 4258 & 530 & 4788 \\
\hline
\end{tabular}

Table 3.

Age-adjusted logistic regression models did not affect outcome measures.

\begin{tabular}{|c|c|c|c|c|}
\hline Internal Injury & Unadjusted Odds Ratio & $95 \%$ Confidence & Limits & P-value* \\
\hline Thoracic & 1.16 & 0.94 & 1.42 & 0.17 \\
\hline Abdominal & 1.57 & 1.23 & 2.01 & 0.0003 \\
\hline Pelvic & 1.53 & 0.72 & 3.29 & 0.87 \\
\hline \multicolumn{5}{|l|}{ Sub Regions } \\
\hline Pulm cont & 1.13 & 0.88 & 1.46 & 0.44 \\
\hline Cardiac & 1.45 & 0.71 & 2.96 & 0.44 \\
\hline Pneumothorax & 0.88 & 0.68 & 1.14 & 0.44 \\
\hline Diaphragm & 0.81 & 0.19 & 3.46 & 0.83 \\
\hline Bronchial tree & 5.37 & 1.51 & 19.14 & 0.04 \\
\hline Aorta & 2.16 & 1.07 & 4.38 & 0.08 \\
\hline Spleen & 1.71 & 1.25 & 2.35 & 0.007 \\
\hline Liver & 1.49 & 1.06 & 2.11 & 0.07 \\
\hline Intestine & 3.99 & 2.28 & 7.09 & 0.001 \\
\hline Bladder & 1.05 & 0.41 & 2.67 & 0.93 \\
\hline Kidney & 1.45 & 0.82 & 2.6 & 0.39 \\
\hline Urethra & 1.3 & 0.55 & 3.1 & 0.65 \\
\hline Pelvic Vasculature & 4.3 & 0.78 & 23.61 & 0.20 \\
\hline
\end{tabular}

${ }^{*}$ P-values for sub-regions are adjusted for multiple testing using Benjamini-Hochberg's False Discovery Rate. 
Table 4.

Open fractures were associated with spleen and liver injury, as well as overall abdominal injury.

\begin{tabular}{ccccc} 
Internal Injury & Odds Ratio & 95\% Confidence Limits & P-value \\
\hline Spleen & 2.30 & 1.11 & 4.75 & 0.04 \\
\hline Liver & 2.36 & 1.07 & 5.23 & 0.04 \\
\hline Abdominal & 2.31 & 1.26 & 4.23 & 0.01 \\
\hline
\end{tabular}

found a statistically significant or borderline significant association with spleen, liver, intestine, and bronchial tree injuries. More internal injuries were observed in open versus closed fractures, and there was no association with shaft versus non-shaft fractures. The findings in open fractures support previous studies relating severity of diaphyseal fractures to concomitant injuries. ${ }^{8}$

It has been shown that motor vehicle accidents in younger individuals occur at higher speeds and energy. ${ }^{9}$ Accordingly, younger patients ( $\leq 18$ years) in this study were most likely to sustain internal injuries. In contrast, of 53 patients $\geq 65$ years with femur fracture, none had internal organ injury. This may relate to a relatively lower energy trauma required to generate a femur fracture in patients' $\geq 65$ years, due to decreasing bone density and reduced load to failure in older age. ${ }^{12}$ In other words, the degree to which older patients are susceptible to femoral fractures could outweigh any increased susceptibility to internal injuries investigated in this study. As such, femoral fracture in ages $\geq 65$ may not be as predictive for associated internal injuries.

Despite improvements in trauma care and vehicle safety standards, fractures of the femur in high-energy trauma continue to cause significant morbidity and resource expenditure. This study clearly demonstrates multiple internal injuries associated with femoral fractures, particularly when open. There is variability in the literature regarding the type of associated injuries. This could be due to multiple factors such as methodology, patient population, changing motor vehicle safety standards or diagnostic sensitivity. Incidence of femur fractures and associated injuries are clearly influenced by motor vehicle safety standards as well as seatbelt laws between different states. With many shifting variables, there is a need for standardization of internal controls regarding specific injuries associated with femoral fractures. Such standardization could possibly be done through radiographic grading of the severity of a femoral fracture and determining the probability of accompanying injuries. ${ }^{8}$

The knowledge of these correlations reaffirms the way we view high impact trauma patients with femoral fractures. However, we do caution that the data are limited and the need for larger multi-center investigations is needed. This study has several strengths. Data were collected from two Level 1 Trauma centers with a large regional network that is representative of large Level 1 Trauma centers. In addition, although a local trauma database was used to initially collect patient encounters, we were able to retrospectively review patient charts to more accurately classify the associated injuries and perform subset analysis. We encourage all providers evaluating patients with femoral shaft fractures in the emergent setting to be aware of these possible associated injuries.

\section{Conflict of Interest: None}

\section{References}

1 Court-Brown CM, Caesar B. Epidemiology of adult fractures: A review. Injury. 2006;37(8):691-7.

2 Weiss RJ, Montgomery SM, Al Dabbagh Z, et al. National data of 6409 Swedish inpatients with femoral shaft fractures: stable incidence between 1998 and 2004. Injury. 2009;40(3):304-8.

3 Lotz JC, Hayes WC. The use of quantitative computed tomography to estimate risk of fracture of the hip from falls. J Bone Joint Surg Am. 1990;72(5):689-700.

4 Adili A, Bhandari M, Lachowski RJ, et al. Organ injuries associated with femoral fractures: implications for severity of injury in motor vehicle collisions. $J$ Trauma. 1999;46(3):386-91.

5 Adili A, Bhandari M, Sprague S, et al. Humeral shaft fractures as predictors of intra-abdominal injury in motor vehicle collision victims. Arch Orthop Trauma Surg. 2002;122(1):5-9.
6 Brahimi H, Rabinovich M, Vuleta V, et al. Biomechanical properties of an intact, injured, repaired, and healed femur: an experimental and computational study. J Mech Behav Biomed Mater. 2012;16:121-35.

7 Rodriguez-Merchan EC, Moraleda L, Gomez-Cardero P. Injuries associated with femoral shaft fractures with special emphasis on occult injuries. Arch Bone Jt Surg. 2013;1(2):59-63.

8 Nikolaou VS, Stengel D, Konings P, et al. Use of femoral shaft fracture classification for predicting the risk of associated injuries. J Orthop Trauma. 2011;25(9):556-9.

9 Kwong Y, Chong M, Hassan A, et al. Severity of injuries associated with femoral fractures as a result of motor vehicle collisions. Arch Orthop Trauma Surg. 2006;126(7):454-7.
10 Scheyerer MJ, Zimmermann SM, Bouaicha S, et al. Location of sternal fractures as a possible marker for associated injuries. Emerg Med Int. 2013;2013:407589.

11 Anandasivam NS, Russo GS, Fischer JM, et al. Analysis of Bony and Internal Organ Injuries Associated With 26,357 Adult Femoral Shaft Fractures and Their Impact on Mortality. Orthopedics. 2017;40(3):e506-e12.

12 Courtney AC, Wachtel EF, Myers ER, et al. Agerelated reductions in the strength of the femur tested in a fall-loading configuration. J Bone Joint Surg Am. 1995;77(3):387-95. 